\title{
La maleta viajera de Euclides, como estrategia didáctica para fortalecer el pensamiento espacial y los sistemas geométricos
}

\author{
Euclid's traveling suitcase, as a didactic strategy to strengthen spatial \\ thinking and geometric systems
}

\author{
Leidy Katerine Marín Arguello \\ a*Especialista en Administración de la informática, 0000-0002-4275-5300, leidykaterinema@gmail.com, Colegio Santos Apóstoles, \\ Cúcuta- Colombia
}

Forma de citar: Marín-Arguello, L.K. La maleta viajera de Euclides, como estrategia didáctica para fortalecer el pensamiento espacial y los sistemas geométricos. Eco Matemático, 10 (1), 76-86

Recibido:19 septiembre 2018

Aceptado: 5 diciembre 2018

\section{Palabras clave}

La Maleta Viajera de Euclides, Estrategia Didáctica, Pensamiento Espacial, Sistemas Geométricos.
Resumen:El desarrollo del pensamiento, incide de manera progresiva en la formación integral de los sujetos, al respecto, se hizo necesario desarrollar la presente investigación, cuyo objetivo general fue: Fortalecer el pensamiento espacial y los sistemas geométricos en los estudiantes de noveno grado del colegio Los Santos Apóstoles mediante la estrategia didáctica, la Maleta Viajera de Euclides, para ello, se adelantó una metodología enmarcada en los postulados de la investigación acción, donde se consideró la puesta en marcha de cuatro fases de manera sistemática, al respecto se asumió la planificación en primer lugar, en esta se diseñaron todos los elementos que contendría la Maleta Viajera de Euclides para el desarrollo del pensamiento espacial y los sistemas geométricos en los estudiantes de grado noveno, seguidamente se presenta la acción, donde se ejecutaron las situaciones diseñadas, en este caso, se evidencia un conjunto de intervenciones, las cuales iniciaron con formas geométricas planas, para posteriormente transformarlas en tridimensionales, para lo cual, los estudiantes representaron disposición e interés en el desarrollo de las actividades, las actividades fueron valoradas mediante la observación directa no participante, para dar paso a la reflexión, donde se logró determinar que la Maleta Viajera de Euclides posee un impacto significativo en la enseñanza y el aprendizaje de los conocimientos inherentes al pensamiento espacial y los sistemas geométricos..

\footnotetext{
*Autor para correspondencia: leidykaterinema@gmail.com
} 


\section{Keywords}

The Euclide Traveling

Suitcase,

Didactic Strategy,

Space Thought,

Geometric Systems.

\section{Introducción}

Los seres humanos día tras día perfeccionan sus acciones, las cuales redundan en la mejora de la calidad de vida, en este sentido, estos en su desarrollo evolutivo, acuden a las entidades educativas con la finalidad de concretar su formación académica, por tanto, se insertan en las mismas y logran la construcción de conocimientos, mediante la práctica constante de elementos que se encuentran implícitos en el currículo, la idea es que el niño y el joven se formen de manera integral para enfrentarse al cambiante mundo de hoy.

Sin embargo, la matemática desde la óptica escolar posee un sinfín de contenidos dentro de su composición curricular, de allí la necesidad de delimitar la investigación en el pensamiento espacial y los sistemas geométricos, dado que los mismos son fundamentales para el desarrollo del pensamiento lógico, porque contribuye con el desarrollo del pensamiento abstracto, dada la complejidad de los sistemas geométricos, es decir, se requiere de elementos que ofrezcan al sujeto elementos necesarios para la consolidación de fenómenos inherentes a la logicidad cognitiva, enmarcada en la producción adecuada de ideas, de acuerdo con lo anterior el Ministerio de Educación Nacional (2015) señala:

El componente geométrico del currículo deberá permitir a los estudiantes examinar y analizar las propiedades de los espacios bidimensional y tridimensional, así como las formas y figuras geométricas que se hallan en ellos. De la misma manera, debe proveerles herramientas tales como el uso de transformaciones, traslaciones y simetrías para analizar situaciones matemáticas. Los estudiantes deberán desarrollar la capacidad de presentar argumentos matemáticos acerca de relaciones geométricas, además de utilizar la visualización, el razonamiento espacial y la modelación geométrica para resolver problemas (p. 12).

Con base en lo anterior, es necesario sostener que desde el currículo, se busca que los estudiantes logren el desarrollo de la capacidad de análisis de las diferentes formas que se encuentran en 
su espacios, tanto de naturaleza bidimensional, como tridimensional, todo ello, contribuye con el desarrollo del pensamiento espacial, es por ello que se considera la necesidad de generar un conjunto de elementos estratégicos que sirvan de base en el desarrollo de este contenido curricular, dichas estrategias deben enfocarse hacia el control de evidencias inherentes a eventos matemáticos.

Bajo el entendido de lo anterior, en la presente investigación, se busca asumir el desarrollo de la Maleta Viajera de Euclides, como una estrategia didáctica para el fortalecimiento del pensamiento espacial y los sistemas geométricos, dicha idea, surge con la intención de motivar a los estudiantes hacia el aprendizaje de la geometría, además de comprometer al docente hacia el desarrollo de fenómenos adecuados al pensamiento espacial, de acuerdo con lo anterior, es necesario destacar que el desarrollo del pensamiento geométrico se logra con la generación de diversas estrategias, dentro de ellas, es necesario asumir la Maleta Viajera, por tanto Pérez (2009) señala:

Partimos en principio de una maleta o portafolios dentro del cual pondremos un cuaderno en blanco y una cinta magnetofónica. Esta maleta va a viajar de casa en casa y se va a ir llenando con la memoria de todos. Su finalidad es recoger muestras de la literatura infantil de tradición oral, es decir todo aquello que los abuelos, los padres o los familiares mayores nos cuenten, reciten o canten a los niños cuando estén jugando con ellos (p. 16).

De acuerdo con lo anterior, la Maleta Viajera, es una estrategia que en principio fue ideada para lengua castellana, dada la versatilidad de la misma, para motivar a los sujetos hacía la generación de aprendizajes significativos, en este sentido, la autora de la presente investigación, consideró necesario su aplicación en el área de matemáticas, en este caso, no se asumirán muestras de literatura infantil, sino de sistemas geométricos que permitan a los estudiantes evidenciar la demanda de acciones presentes en la realidad, es decir, desde la concreción de elementos materiales y estratégicos para el desarrollo de contenidos inherentes a la geometría propiamente dicha, en este plano, es necesario reconocer la demanda de los sujetos, en relación a situaciones que activen su creatividad, para de esa manera fortalecer su estructura cognitiva.

Esta estrategia busca también incorporar a los padres de familia y a la comunidad en general para la formación de los estudiantes, lo más importante en este caso, es la naturaleza viajera de la estrategia para de esa manera lograr la motivación de los estudiantes y de todos los responsables del proceso de la enseñanza y el aprendizaje, en este caso, es necesario manifestar el interés de los sujetos en relación a situaciones inherentes al desarrollo de competencias geométricas, donde mediante la construcción de situaciones que se promuevan en el aula de clase, se logre la construcción de aprendizajes significativos.

Por tanto, se desarrolló una metodología amparada en el paradigma cualitativo, desde la perspectiva de la investigación acción, la misma busca la comprobación de la solución en la realidad, para la cual fue determinada, de allí la necesidad de sistematizar el estudio en una serie de pasos que sirvieron de base para la puesta en marcha del método científico, donde se partió de la definición del problema, los objetivos del estudio y la justificación, un segundo momento que hace énfasis en la adopción de los elementos teóricos que fundamentan el estudio, seguidamente se presenta la metodología de la investigación. Seguidamente se presenta el cuarto capítulo, donde se presentan de manera organizada la propuesta pedagógica.

\section{Materiales y métodos}

Se trabajó con la investigación acción la cual responde en gran medida a la naturaleza particular del estudio a investigar, el cual plantea la incorporación activa de los implicados en el estudio y actúa frente a las realidades sociales en una constante interacción reflexión-acción. Este 
método es visto por Cendales (2007) como “... una propuesta metodológica que forma parte de una estrategia que involucra a la comunidad en el conocimiento y solución de sus problemas" (p.41). Con estos señalamientos, se demuestra el proceso vivencial y la pertinencia de la Investigación Acción (IA), puesto que, permite percibir directamente los comportamientos y actitudes de los individuos en el proceso de aprendizaje, mediante un ciclo que se asume desde la planeación, la acción, la observación y la reflexión, para de esa manera contar con situaciones pertinentes acerca de las intervenciones realizadas.

De esta manera, la IA se propone concientizar e identificar al investigador con el estudio desarrollado, por lo que en el marco de este planteamiento fue de utilidad, por cuanto se determinó con certeza la intervención de los docentes en el desarrollo del pensamiento espacial y el proceso de enseñanza y aprendizaje de los estudiantes en torno a este y a los elementos geométricos. A tal efecto, este enfoque sirvió de herramienta para interactuar y reflexionar ante los encuentros y hallazgos logrados, lo que implicó captar las expresiones y motivación de los docentes y estudiantes ante la intención del estudio.

A lo anterior se agrega, los señalamientos realizados por Caricote (2008) al indicar que la investigación acción contribuye a:

Elevar el nivel intelectual de los participantes, proporcionando instrumentos de participación y capacitación... ella permite sistematizar las experiencias populares, pero también democratizar el saber y fortalecer la organización de la propia comunidad en función de sus proyectos políticos. Se trata de un modelo de sociedad y de conocimiento realmente democrático y participativo. (p.97).

Tales apreciaciones demuestran el procedimiento reflexivo, sistemático, organizado $\mathrm{y}$ crítico de la Investigación Acción, lo que permite a los miembros de una comunidad educativa convertirse en protagonistas del proceso para detectar problemas, necesidades e intereses y así dar propuestas y soluciones, lo cual significa que el modo de realizar el estudio es a través de la intervención y propósito está vinculado a la acción.

Se considera pertinente complementar la naturaleza metodológica de este trabajo, debido a la intención de profundizar en las múltiples dimensiones que representa el pensamiento espacial y los sistemas geométricos con el carácter de un estudio de campo, puesto que, se hace necesario estar en el contexto para complementar la adopción didáctica. Para Arias (2010) la investigación de campo consiste en "caracterizar un hecho, fenómeno o grupo con la finalidad de establecer su estructura o comportamiento" (p.46). Asimismo, la Universidad Pedagógica Experimental Libertador (2008) la plantea como “...el análisis sistemático de problemas en la realidad, con el propósito bien sea de describirlos, interpretarlos, entender su naturaleza o predecir su ocurrencia" (p.18).

Es decir, se recoge los datos directamente donde ocurren los hechos de manera natural, lo que permitirá a la investigadora el análisis de los comportamientos y actitudes frente al desarrollo del pensamiento espacial y los sistemas geométricos. Conviene destacar, más que demostrar el comportamiento de los docentes y de los estudiantes, con base a las acciones manejadas en la investigación.

\section{Fases de la Investigación}

Dada la naturaleza de la investigación acción, es pertinente el desarrollo de una serie de fases que permitirán el desarrollo de la misma, al respecto Hurtado (2007) plantea:

Fase I Planificación: Una vez identificado el problema, en el diagnóstico, se procedió con la fijación de elementos necesarios a incorporar en la Maleta Viajera de Euclides, es decir a las intervenciones pedagógicas que promueven el desarrollo del pensamiento espacial y los sistemas geométricos en los estudiantes de grado noveno. 
Fase II Acción: En este caso, se consideró la aplicación de las intervenciones pedagógicas en el aula de clase, como base de desarrollo del pensamiento espacial y los sistemas geométricos en los estudiantes de grado noveno, a partir de la Maleta Viajera de Euclides.

Fase III Observación: A la par de la fase anterior, en la ejecución de cada de las acciones, se hizo necesaria la aplicación de la observación, para recolectar los datos que permitieran definir situaciones propias de la Maleta de Euclides.

Fase IV Reflexión:Se confrontó la realidad observada, para de esta manera hacer los ajustes a la realidad y contar, así como elementos necesarios para la generación de conocimientos científicos.

\section{Descripción del Escenario}

El contexto físico en el cual se desarrolló el estudio, fue la Institución Educativa Santos Apóstoles, liderada actualmente por el Especialista Otardo Rincón Contreras, fue fundado por el Pbro. Jaime Montagut Vega con Acto Legislativo No 0229 del 10 de marzo de 1994. Se encuentra ubicada en la Avenida 6 NN $^{\circ}$ 6-54 del barrio Chapinero, de la ciudad de Cúcuta, es de clase oficial y carácter mixto, jornada mañana continua y tarde continua; que ofrece educación formal en los niveles: Preescolar (grado transición), básica: primaria (grados 1, 2, 3, 4 y 5), secundaria (grados 6, 7, 8 y 9), Media Académica (grados 10 y 11). En donde asisten aproximadamente unos 3500 niños.

La población está distribuida en cinco sedes, cuatro de primaria que fueron fusionadas con el colegio Santos Apóstoles, con el desarrollo de la Ley 715 del 21 de Diciembre de 2001, emanada del Ministerio de Educación Nacional que reglamenta $\mathrm{y}$ orienta el nuevo funcionamiento y accionar de las Instituciones educativas, se crea la fusión mediante Decreto No 00081 del 30 de Septiembre de 2002 de los siguientes establecimientos educativos: La
Escuela Urbana de Niñas, Escuela Kennedy $N^{\circ} 47$, Escuela El Rosal, La Escuela José Celestino Mutis, La No 19.

\section{Población y Muestra}

En lo concerniente al estudio, que se refiere a diagnosticar las estrategias didácticas empleadas en el fortalecimiento del pensamiento espacial y los sistemas geométricos en el grado noveno, requiere de la selección de informante claves, los cuales constituyó fuentes primarias implicadas en la investigación. Al respecto Rodríguez, Gil y García (ob. cit) expresan:

... los informantes, participantes o sujetos de investigación resultan elementos imprescindibles. Ellos aportan la mayor parte de la información primaria sobre el problema de investigación. Son las personas a quienes con más frecuencia se observa, pregunta (...) tienen acceso a la información más importantes sobre las actividades de una comunidad (...) es un verdadero guía que enseña al investigador a enfrentarse con la comprensión de un problema. (p.127).

De acuerdo con los señalamientos de los autores antes mencionados, estos informantes claves resultan elementos imprescindibles para recoger la información necesaria de acuerdo con la naturaleza del estudio. Por lo tanto, además de las condiciones descritas con anterioridad, fueron seleccionados mediante los siguientes criterios, (a) docentes y estudiantes, (c) conocedor de las estrategias didácticas para el desarrollo del pensamiento espacial, (d) disposición para brindar la información necesaria de forma voluntaria. De este modo, se escogieron estos informantes quienes cumplieron con características especiales, puesto que, constituyen actores educativos y forman parte de la dinámica escolar inmersa directamente en las aulas de clase. Tomando en cuenta estas características, la población fue de 55 estudiantes del grado noveno uno de la jornada de la mañana de la referida institución. 
Estas personas fueron los actores principales que proyectaron sus intereses compartidos, inquietudes $\mathrm{y}$ argumentos en las vivencias planteadas, de los cuales emergió el análisis de la información.

\section{Técnicas e Instrumentos de Información}

Con el propósito de alcanzar los objetivos de la investigación, referente a el diagnostico de las estrategias didácticas empleadas en el fortalecimiento del pensamiento espacial y los sistemas geométricos en el grado noveno, se propone la observación participante apoyada en los diarios pedagógicos, lo que implicará que la investigadora esté conectada directamente con el aula de clase, es decir, requiere de involucrarse y hacerse participe de la vida escolar y acciones propias de los docentes y estudiantes. Para Hurtado (2007) “...la observación participante implica que el investigador se introduzca en la comunidad o grupo que se propone estudiar". (p.110). Es decir, fue necesario estar inmerso en las acciones de los docentes es estudiantes referentes a las estrategias didácticas empleadas en el desarrollo $\mathrm{y}$ fortalecimiento del pensamiento espacial $\mathrm{y}$ sistemas geométricos y sus implicaciones.

\section{Análisis de los Datos}

Debido a lo riguroso de la labor que resulta el análisis de la información, y que además se logró recaudar la información necesaria surgida de las interacciones con los sujetos y sus opiniones dadas durante el curso de la investigación, el análisis de este trabajo estuvo dirigido a escudriñar rigurosamente y sistemáticamente un grupo de elementos informativos, claro está, preservando su naturaleza y fiabilidad de los mismos, que requiere de sintetizar categorizar y estructurar para dar sentido, coherencia y comprensión a la investigación planteada. En tal sentido, para Rodríguez, Gil y García (et. al) define el análisis de datos como: “... uno conjunto de manipulaciones, transformaciones, operaciones, reflexiones, comprobaciones que realizamos sobre los datos con el fin de extraer significado relevante en relación a un problema de investigación" (p.200). A partir de esta premisa, se condujo a dar una visión de los innumerables puntos de vista de las personas implicadas en el estudio, los cuales expresaran sus intereses, molestias, deseos y aportes a la solución del problema.

A tal efecto, los datos conllevaran a utilizar y aplicar la teoría fundamentada de Strauss y Corbin citados Rodríguez, Gil y García (et. al) la cual se basa en un método de análisis cualitativo que consiste en la codificación dada por el investigador a través de palabras de cada uno de los entrevistados, así como las incidencias ocurridas en la realidad para luego ser agruparlos en categorías, conceptos o constructos para establecer las diferencias y semejanzas con respecto a una u otra categoría que el investigador identifique (p.48). Se procedió a aplicar el proceso de análisis de datos cualitativos en la reducción de los mismos a categorías primarias para luego ser deducidos por indicadores. Seguidamente para darle sentido a los datos obtenidos se procedió a realizar una relación entre categorías primarias y los indicadores. Asimismo, para mayor comprensión de los resultados se sintetizará de forma gráfica, al respecto, se evidenciarán los presaberes de los estudiantes, en relación con los indicadores de pensamiento espacial y los sistemas geométricos.

\section{Resultados y análisis}

Los resultados surgieron de la aplicación de los diarios de campo para el diagnóstico, dada la naturaleza de la investigación acción, se aplicó la observación directa, la cual permitió establecer una serie de situaciones inherentes a la contextualización de los elementos propios de la realidad, en este sentido, se asumieron como categorías de análisis: Metodológica, didáctica, pragmática y contextual, estas categorías permitieron evidenciar el desarrollo de las clases desde cada una de ellas, para de esta forma lograr tener una visión global de la realidad estudiada, y verificar que la propuesta pedagógica a poner en práctica fuese la más adecuada. 
Por tanto, es necesario establecer que los resultados se generaron con base en los diagnósticos, de acuerdo con la ejecución de la observación directa, no participante, la misma es definida por Hurtado (2007) como: "la observación directa no participante, se define mediante las acciones que el investigador ejecuta, para lograr la captación de los eventos de su interés, se trata de no incidir en el desarrollo de las acciones, solo de registrar el evento" (p. 123), en la ejecución de la observación, se acudió a esta técnica debido a que se asume como base la investigación acción para tal fin, es importante aclarar que en la observación, también se desarrollaron diálogos informales, pero debido a esa informalidad, no se incluyeron en la investigación, porque pierde de vista la cientificidad del estudio, en este sentido, es pertinente rescatar los siguientes aspectos, inherentes al desarrollo de situaciones propias de la realidad observada:

Los estudiantes inicialmente no demostraban interés por los sistemas geométricos, de igual manera se logró establecer que el desarrollo del pensamiento espacial, en la mayoría de los casos era escaso y en otros nulo, realmente preocupante la situación, dado que el poco interés de los estudiantes por las clases de matemática no permita la aplicación de alguna motivación que los impactará de manera efectiva, por el contrario, se evidenciaba descontento, desinterés, apatía, decidía, entre otras características poco adecuadas que pudieran generar algún conocimiento al respecto.

En el mismo orden de ideas, los estudiantes se muestran indispuestos frente a las clases de matemática, de igual manera no les gusta participar en esta clase, es decir, se muestran desmotivados, frente a la enseñanza de elementos geométricos, en algunos casos se observa sueño, en algunos otros, desgano, pereza, no se demostraba una disposición clara hacia la clase, por el contrario el desagrado frente a la misma era muy marcado, en este caso, es preciso hacer referencia a los señalado por Hernández (2006):
La enseñanza de las ciencias exactas, es cada día más compleja, a medida de que aumenta el uso de la tecnología, los jóvenes pierden el interés por asistir a las instituciones educativas y permanecer horas en una aula de clase sentados, este es el panorama, en la enseñanza de la matemática, la química, la física, entre otros, lo cual, está generando fracasos en los jóvenes y en la misma calidad de la educación ( $\mathrm{p}$. 29).

De manera que el desagrado por la enseñanza de las matemáticas es notorio en los estudiantes, los mismos prefieren algunos otros elementos externos, antes que disponer de su actitud para el desarrollo de la clase de matemática. De igual manera, se logró establecer que los estudiantes carecen de compromiso, cuando no presentan las actividades asignadas para la casa, es caso perdido colocar una asignación para el hogar y cuando se cita al representante para que indique por qué su hijo no está cumpliendo con dichas asignaciones, estos dicen estar muy ocupados, como para encargarse de la formación de sus hijos, lo cual es lamentable porque el trabajo que se desarrolla en la institución educativa, no se respalda en el hogar, poniendo en riesgo el trabajo docente.

Asimismo, no llevan los materiales necesarios para la institución educativa, lo cual no permite el desarrollo adecuado de las actividades planificadas por la docente, cuando se les solicita juego de geometría, la mayoría no lo posee, en algunos casos, ni lápiz poseen para registrar las actividades, son muy pocos los estudiantes que llevan los materiales, menos aún, cuando se les solicita un material para un trabajo práctico, dicen haberlo olvidado, o que los padres no tenían el dinero suficiente para adquirirlo, o que simplemente no lo llevaron porque no lo disponen así.

Las clases de matemática son monótonas, no se refleja el interés de los estudiantes, por el contrario, es notorio el rechazo hacia el área de matemática, debido a ello, el desarrollo de contenidos 
relacionados con el pensamiento espacial es de muy difícil acceso para los estudiantes, en ello, se refleja el escaso conocimiento geométrico que poseen los estudiantes, lo cual, entorpece el desarrollo de los contenidos relacionados con la geometría, sin embargo, la investigadora como docente ha tratado de hacer clases amenas, las cuales son rechazadas por los estudiantes, diciendo que: "la matemática no es importante", "yo no voy a estudiar una carrera donde use matemática", en fin estas aseveraciones, hacen que la situación en muchos momentos se torne insostenible.

Finalmente, los estudiantes no comprenden el dominio de las áreas y segmentos, así como de los indicadores del conocimiento espacial, no se desarrollan, alterando la prosecución conceptual necesaria para la constitución de una estructura cognitiva, encaminada a dar respaldo a las acciones del estudiante para la vida.

Una vez generada la revisión del diagnóstico previo, es necesario destacar que se requiere de un mecanismo didáctico que sirva de base en la dinamización de las clases de matemática, específicamente en las relacionadas con el pensamiento espacial y los sistemas geométricos, por tanto, se manifiesta el interés de la investigadora por fortalecer el pensamiento espacial y los sistemas geométricos en los estudiantes de noveno grado del colegio Los Santos Apóstoles mediante la estrategia didáctica, la Maleta Viajera de Euclides, con miras a superar las situaciones inherentes a la desmotivación inicialmente y al rechazo por la clase de matemática, lo cual es urgente, para de esa manera lograr un desarrollo adecuado de las mismas, al respecto, es necesario generar la propuesta pedagógica.

Una vez presentadas las evidencias, es necesario destacar que en la categoría metodología, se establece el nivel de conocimientos de los estudiantes, por ello, fue necesario tomar los postulados de Zabala (2012) inherentes a la discusión dirigida, como una de las estrategias que dio un resultado esperado, por tanto, la investigadora, establece como parte fundamental la construcción de conocimientos mediante esta estrategia. De igual manera, se establecieron los ejercicios de representación visual, muy importantes para la construcción de aprendizajes significativos, esto se encuentra sustentado en Godino y Batanero, además de ello, la autora considera que para que haya un amplio reconocimiento de las figuras planas, se requiere de ejercicios de representación visual.

En el mismo orden de ideas, es necesario reconocer el trabajo de los estudiantes en relación con la construcción de las figuras geométricas, esto sustentado en Aguirre (2010) y en lo que la investigadora desarrollo en el aula de clase, mediante el empleo del trabajo práctico, con la elaboración de los cilindros. De igual manera, la construcción de esferas, como lo manifiesta Godino y Batanero (2003), lo cual, se evidencia en la realidad, a pesar de la complejidad observada, la investigadora considera necesario asumir las esferas para de esta manera lograr un aprendizaje significativo. Los sistemas geométricos y su enseñanza son complejos, así como lo indica Godino y Batanero (2003), por ello, es necesario la motivación de los estudiantes, así como se logró establecer en los hallazgos y en lo que manifestó la investigadora en la realidad.

Con respecto a la categoría didáctica, se logró establecer desde la teoría la importancia de la enseñanza de las proporciones en la geometría, por lo cual, en la realidad se estableció la resolución de problemas, situación que la investigadora considera fundamental para el desarrollo de tal fin. Por su parte, en relación con la enseñanza del área Godino y Batanero (2003), manifiestan su importancia, por lo cual, se hizo necesaria la generación de conceptos propios en relación con ello, para de esa manera asumir el hecho de los presaberes de los estudiantes y la valoración de los mismos.

En el mismo orden de ideas, el empleo de imágenes, permiten poner en evidencia la estrategia de representación, lo cual, se logró en la realidad 
y a juicio de la investigadora es fundamental, para el logro de evidencias necesarias en la realidad. Aunado a lo anterior se presentan las estrategias de demostración, presentadas por Díaz (2007) y las cuales como hallazgos dieron un resultado significativo y en la realidad la investigadora asume las mismas como base en la didáctica de los sistemas geométricos de representación. De igual manera, se manifiestan evidencias en elaborar un trabajo que de resultado como lo manifiesta Díaz (2007), de igual manera, los estudiantes consideran que los ejercicios prácticos son fundamentales, así como la investigadora considera que su impacto es favorable en la enseñanza del pensamiento espacial.

En el caso de la categoría pragmática, se manifiesta el establecimiento de semejanzas, propuesto por Brousseau (2006), donde se logró poner en evidencia la capacidad de los estudiantes para tal fin, así como la investigadora, logró establecer la necesidad de estos elementos. De igual manera, se presenta el uso del tablero, atendiendo a los postulados de Díaz (2007) y mediante las comprobaciones en la realidad, desde el trabajo con los docentes y las situaciones establecidas por la investigadora, en el desarrollo de la investigación, se logró denotar la importancia del trabajo práctico, propuesto por Díaz (2007) donde permitieron evidenciar la disposición de los estudiantes hacia tal fin.

\section{Conclusiones}

Las conclusiones son el fundamento en el desarrollo de toda investigación, dado que las mismas permiten fijar las respuestas a los objetivos planteados en el estudio, en este sentido, es necesario partir por el objetivo general, en el cual se planteó: Fortalecer el pensamiento espacial y los sistemas geométricos en los estudiantes de noveno grado del colegio Los Santos Apóstoles mediante la estrategia didáctica, la Maleta Viajera de Euclides.

En relación con el objetivo específico número uno: Diagnosticar las estrategias didácticas empleadas en el fortalecimiento del pensamiento espacial y los sistemas geométricos en el grado noveno, se logró detectar de que a pesar de que la docente emplea las estrategias de representación en el tablero y de motivación, las mismas no son efectivas, dado que los estudiantes rechazaban con regularidad el área de matemática, es decir no demostraban ningún interés relacionado con tales situaciones, lo cual, reflejaba la urgente necesidad de incorporar elementos que dinamizaran tanto el proceso de enseñanza, como de aprendizaje.

En relación con el segundo objetivo específico: Evidenciar los presaberes de los estudiantes de grado noveno sobre los temas geométricos a contener en la Maleta de Viajera de Euclides, en este caso, se fijaron como elementos fundamentales las figura planes geométricas y sus componentes, desde la consideración del área para tal fin, de igual manera, se asumieron los elementos inherentes a la transformación de las figuras planas en tridimensionales, para la constitución adecuada de los presaberes

En cuanto al tercer objetivo específico: Realizar la intervención didáctica denominada la Maleta Viajera de Euclides, dicha estrategia se puso en marcha, mediante la aplicación sistemática de cinco intervenciones, inicialmente se evidenció como los estudiantes se mostraron curiosos y querían que se les indicará en qué consistía el uso de la Maleta Viajera, lo cual fue gratificante porque de entrada e logró poner en evidencia el interés de los estudiantes, posterior a ello, se logró la motivación de los mismos, estos querían intervenir de manera activa en todas las actividades, en este caso, es necesario destacar que esta estrategia, posee una naturaleza inclusiva, es decir, se involucra a todos los alumnos por igual y se le presta atención a aquellos que poseen problemas de comprensión de los ejercicios, para que de esta manera se logre un aprendizaje generalizado en el grupo de estudiantes, de igual manera se elevó el interés de los mismos en relación con la clase de matemática, en general, 
porque los estudiantes estuvieron de acuerdo en que se manejará la Maleta para los contenidos a que hubiere lugar, con la final de motivarlos hacia el aprendizaje.

En cuanto al cuarto objetivo específico: Reflexionar sobre el impacto de la Maleta Viajera de Euclides en el fortalecimiento del pensamiento espacial y los sistemas geométricos, es necesario establecer que esta estrategia conduce a elementos fundamentales dentro del aula de clase, es relevante observar como los estudiantes cambiaron su percepción en relación con la matemática, de igual manera el rendimiento académico ha sido uno de los factores más favorables, puesto que desde el mismo se evidencia como ha subido y se ha perfeccionado, los conocimientos de los estudiantes a la fecha son significativos y cuenta con la aceptación de la comunidad en general.

En este sentido, es necesario destacar que se concreta una estrategia significativa en el desarrollo del pensamiento espacial de los estudiantes, por tanto, se hizo necesario la adopción de la misma como una estrategia que dinamice y oriente el desarrollo de las clases de matemática, todo ello, centrados en la construcción de aprendizajes significativos, donde el trabajo del estudiante sea el que tome el rumbo para el establecimiento de saberes inherentes a la consolidación de la estructura cognitiva de los sujetos, es así, como la Maleta Viajera de Euclides, no sólo incide en la motivación de los estudiantes, sino que se concreta en función de la calidad educativa del país, brindando acciones que se manifiesten desde el aula de clase.

\section{Referencias}

Aguirre, J. (2010). Habilidades Matemáticas desde la Práctica de la Música. Trabajo de Grado de Maestría. Universidad Autónoma de Monterrey. México

Arias, F (2010). El Proyecto De Investigación Introducción A La Metodología Científica. Caracas, Venezuela: Episteme.
Brousseau (2006). Enseñanza de la Matemática. Mac Graw Hill ediciones interamericana. México

Caricote, M. (2008). Métodos de Investigación en Ciencias Sociales. Ediciones Siglo XXI. Argentina.

Cendales, O. (2007). Investigación Acción y Saber Social. Editorial Grao. España.

Díaz, F. (2007). Estrategias de Supe aprendizaje. Editorial Trillas. México

Fabretti, I. (2009). Estrategias Didácticas. Editorial Gedisa. España.

Fairstein y Gissels (2004). Matemática y su Enseñanza. Editorial Paraninfo. Argentina

Godino, J. y Batanero, F. (2003) Geometría Y Su Didáctica Para Maestros. Departamento de Didáctica de la Matemática. Universidad de Granada. Granada

González, M. (2005), Estrategias Didácticas. Ediciones El Nacional. Caracas.

Gutiérrez B. L. (1989). Paradigmas cuantitativo y cualitativo en la investigación socio-educativa: Proyección y reflexiones. Revista Paradigma, Vol. XIV al XVII, 1993 - 1996. [Documento en línea] http://www.perio.unlp.edu.ar/Metodologia/ texparabajar/locualiylocuantilgutierrez1.doc. [Consulta 2015. Marzo 08].

Hernández, D. (2006). Enseñanza y Aprendizaje. El Reto Social. Ediciones Aljibe. España.

Hurtado, J. (2007). La Investigación Holistica. Sypal. Venezuela

Klein, M. (2008). Psicoanálisis de Niños. Editorial Paidos. España.

Luengo, M. (2011). Formación didáctica para profesores de matemáticas. Madrid: Editorial CCS

Martínez y Álvarez (2013). Creación de ambientes de aprendizaje en la enseñanza de polígonos; una experiencia de aula desde la educación matemática crítica

Mena, C. (2008). Estrategias de Enseñanza en la Escuela. Ediciones Oceano. Colombia

Ministerio de Educación Nacional (2010). Pruebas Saber Pruebas Saber $3^{\circ}, 5^{\circ}$ y $9^{\circ}$. Documento en Línea. Disponible en: http://www.mineducacion. 
gov.co/1759/w3-article-244735.html Fecha de consulta: 03 de enero de 2017.

Ministerio de Educación Nacional (2015). Documento orientador - Foro Educativo Nacional 2015: ciudadanos matemáticamente competentes

Morgado, M. (2001). Concepciones Pedagógicas de la enseñanza. Editorial Trillas. México

Mûnch y Ángeles (2004). Metodología de la Investigación. Editorial Limusa. México

Núñez, J. (2006). Investigación Acción. FEDUPEL. Venezuela.

Ortiz, P. (2009). El Currículo Oficial. Ediciones Piramidal. Argentina.

Pérez, C. (2009). La Maleta Viajera. Universidad de Malaga.

Ramírez y Chavarría (2009). Análisis de las conceptualizaciones erróneas en conceptos de geometría y sistemas de ecuaciones: un estudio con estudiantes universitarios de primer ingreso

Rodríguez, Gil y García (1999). Metodología de la Investigación. Mac Graw Hill ediciones Interamericana

Ruíz, M. (2008). Teorías y Enfoques de Aprendizaje. Ediciones Norma. colombia

Vasco, H. (2010). Enseñanza de la Geometría en la Escuela. Editorial Gedisa.

Viera, A. (2008). Matemáticas y medio: Ideas para favorecer el desarrollo cognitivo infantil. Sevilla: DIADA Editora

Zabala, P. (2012). Estrategias para la Enseñanza y el Aprendizaje. Editorial Trillas. México

Zambrano, D (2010). Conocimiento, saber y pensamiento: una aproximación a la didáctica de las matemáticas. EquisAngulo. Colombia 